Editorial

\title{
Interventional Radiology Training in India
}

\author{
Shyamkumar N. Keshava ${ }^{1}$ Sanjeeva Kalva ${ }^{2}$ \\ ${ }^{1}$ Department of Radiology, Christian Medical College, Vellore, Tamil \\ Nadu, India \\ 2 Division of Interventional Radiology, Department of Radiology, \\ University of Texas Southwestern Medical Center, Dallas, TX, USA
}

J Clin Interv Radiol ISVIR 2017;1:67.

Significant advances in interventional radiology (IR)and image-guided therapies during the recent years have resulted in the need of specialized and advanced training for medical graduates interested in the practice of this specialty. While the formal training in IR is still part of the 3 -year postgraduate course in diagnostic radiology, it is, at best, heterogeneous and limited to a few centers. The increasing breadth of diagnostic radiology and lack of formal advanced training courses in radiologic subspecialties following the postgraduate course have reduced the focus on IR training during the postgraduate course. At best, most trainees are left with limited experience in ultrasound and computed tomography (CT)-guided biopsies and drainage procedures and rarely have mastered the art of practicing clinical interventional radiology.

The practice of IR requires adequate training in the interpretation of imaging studies, including ultrasound, CT and magnetic resonance imaging (MRI); procedural skills to perform both vascular and nonvascular interventions; and medical management of patients prior to, during, and after interventional radiologic procedures. Noninterpretive skills in professionalism, ethics, and cost-effectiveness; training in research methodology; clinical collaboration across various medical and surgical specialties; and knowledge of judicial aspects related to IR would improve the chances of success during practice of IR. In addition, facilities that encourage and provide opportunities for teaching junior residents and medical students and active participation in national and international conferences would provide a more rewarding experience during IR training.

While a standardized curriculum is of paramount importance for training in IR, not less important are the standardized evaluation of trainees and accreditation of centers of training for adequate equipment and facilities required for such training. The efforts of the Indian Society of Vascular and Interventional Radiology (ISVIR) in this endeavor are commendable. The leaders and volunteers of ISVIR have worked effortlessly to bring training courses into fruition. The IR training is considered a super-specialty course with an award of "Doctor in Medicine (DM) in Interventional Radiology" after successful completion of training and posttraining examinations. The course is for 3 years and students are selected after successful completion of a 3-year postgraduate course in diagnostic radiology through an entrance examination. The National Board (NB) provides opportunities to start training a 3-year FNB (Fellowship in National board) course in IR that would still be accredited and supported through an independent posttraining certifying examination.

These changes to the training of IR in India are still at starting level but are timely and would ensure adequately trained interventional radiologists in the workforce. In addition, IR would prosper as a designated super-specialty and allow improved collaboration across the different clinical specialties.
Address for correspondence Shyamkumar N. Keshava, DMRD, DNB, FRCR, FRANZCR,

Department of Radiology, Christian Medical College, Ida Scudder Road, Vellore 632004, Tamil Nadu, India (e-mail: aparna_shyam@yahoo. com).
DOI https://doi.org/

$10.1055 / \mathrm{s}-0037-1603531$. ISSN 0000-0000.
Copyright ( 2017 by Indian Society of Vascular and Interventional Radiology
License terms

()(1) $\Theta \circledast$ 